\title{
Accurate Evaluation of Polarization Characteristics in the Integrated Optic Chip for Interferometric Fiber Optic Gyroscope Based on Path-matched Interferometry
}

\author{
Woo-Seok Choi* and Min-Sik Jo \\ The 3rd R\&D Institute, Agency for Defense Development, Yuseong P.O. Box 35-3, \\ Daejeon 305-600, Korea
}

(Received March 19, 2009 : revised July 1, 2009 : accepted July 1, 2009)

\begin{abstract}
Accurate evaluation of polarization characteristics in the integrated optic chip (IOC) for interferometric fiber optic gyroscope was performed. Spatial distribution of optical wavetrains caused by the polarization parameters such as local polarization cross-coupling and polarization rejection coefficient of the IOC were measured utilizing the path-matched optical coherence domain polarimetry (PM-OCDP). With the analytic model deduced from Jones matrix representation, we could accurately identify the polarization characteristics of the IOC. Both degree of measurement error due to the imperfect equipment conditions in PM-OCDP and birefringence of IOC chip were also characterized.
\end{abstract}

Keywords : Fiber optic gyroscope, Integrated optic chip, Optical coherence domain polarimetry OCIS codes : (060.2800) Gyroscopes; (060.2370) Fiber optics sensors; (130.3120) Integrated optics devices; (230.5440) Polarization-sensitive devices

\section{INTRODUCTION}

Interferometric fiber optic gyroscope (IFOG) is one of the representative optical rotation sensors based on the Sagnac effect $[1,2]$. By now, IFOG have matured technically with rapid development of devices for optical communications and have become a good substitute for the other traditional gyroscopes. In the development of navigation grade IFOG, it is necessary to pay attention to the birefringence induced polarization non-reciprocity caused by the considerable influence of imperfect polarization characteristics between constitutive optical components on the bias stability [3]. The related theories have been well established and many solutions have been suggested utilizing a polarizer with the common input/output port of the Sagnac closed loop [4-6]. Intensity-typed phase error due to the interference between the main polarization mode and the crosscoupled secondary polarization mode is bounded by the value proportional to the square of the amplitude extinction coefficient of the polarizer as follows [7],

$$
\left|\Delta \phi_{e}\right|<\rho_{r}^{2} \varepsilon^{2}
$$

\footnotetext{
*Corresponding author: cws@add.re.kr
}

where $\Delta \phi_{e}$ is intensity-typed phase error due to the polarization cross-coupling, $\varepsilon$ is the amplitude extinction coefficient of the polarizer, $\rho_{r}^{2}$ is the intensity ratio of the cross-coupled mode to the main polarization mode. In addition, amplitude-typed phase error due to the interference between the cross-coupled mode from the secondary mode and the main mode is limited by the value proportional to the amplitude extinction coefficient of the polarizer as follows,

$$
\left|\Delta \phi_{e}^{\prime}\right|<2 \sqrt{\rho_{i}} \rho_{r} \varepsilon
$$

where $\Delta \phi_{e}{ }^{\prime}$ is amplitude typed phase error due to polarization cross-coupling, $\rho_{i}$ is the intensity ratio of the secondary mode over the main mode of the optical wave propagating to the input port in front of the polarizer. In any case, the polarization extinction coefficient of the polarizer is a very critical factor influencing the birefringence induced bias instability.

Since the 1980's a striking development of integrated optics utilizing matured semiconductor fabrication processes with the advantages of compactness, robustness and cost-effectiveness have been also applied to IFOG and been instrumental in the development process of IFOG [8]. The integrated optic chip (IOC), a device with the three key functions of Y-beam splitter, electro- 


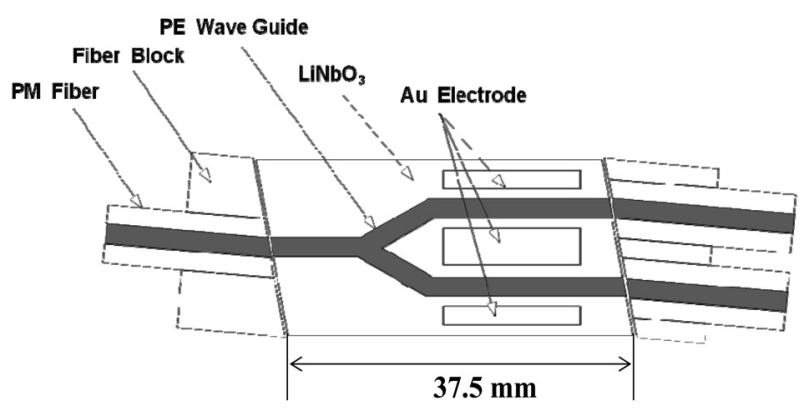

FIG. 1. Schematic view of IOC for IFOG.

optical phase modulator and polarizer, is one of essential parts of IFOG. A schematic view of the IOC is shown in Fig. 1. The strong birefringence of protonexchanged $\mathrm{LiNbO}_{3}$ optical waveguide proves to be very satisfactory as a transverse magnetic field (TM) mode rejecting polarizer with very low extinction coefficient below $-50 \mathrm{~dB}$ [9]. As stated earlier the polarization characteristics such as polarization extinction and local polarization cross-coupling in the Sagnac closed loop are critical factors in determining IFOG bias performances. So it is very important to get accurate characterization of such factors for systematic performance improvement of IFOG.

In this study, accurate evaluation of polarization characteristics in the IOC for IFOG was performed. With the path matched-optical coherence domain polarimetry (PM-OCDP) and analytic formalism utilizing Jones matrix representation, we could accurately identify the polarization characteristics of the IOC in terms of the $\rho_{r}$ and $\varepsilon$.

\section{MEASUREMENT OF POLARIZATION CHARACTERISTICS BASED ON PM-OCDP}

Generally, polarization characteristics of optical devices can be measured by passing light from an unpolarized broadband source through them and analyzing the intensity ratio between the two orthogonal polarization modes utilizing a polarizer [10]. However, in the case of an in-line component such as the IOC, this kind of methodology can't be applied for two reasons. One is because of hybrid bonding points between two different substances, for example, $\mathrm{LiNbO}_{3}$ chip and input/output pigtailed polarization maintaining fiber (PMF), there exist at least two local polarization cross-coupling points. So it is impossible to discriminate any one of the local polarization cross-couplings from another local polarization cross-coupling or from polarization extinction. Another reason is related to finite polarization rejection of the analyzing polarizer. If the measured sample has a lower extinction coefficient

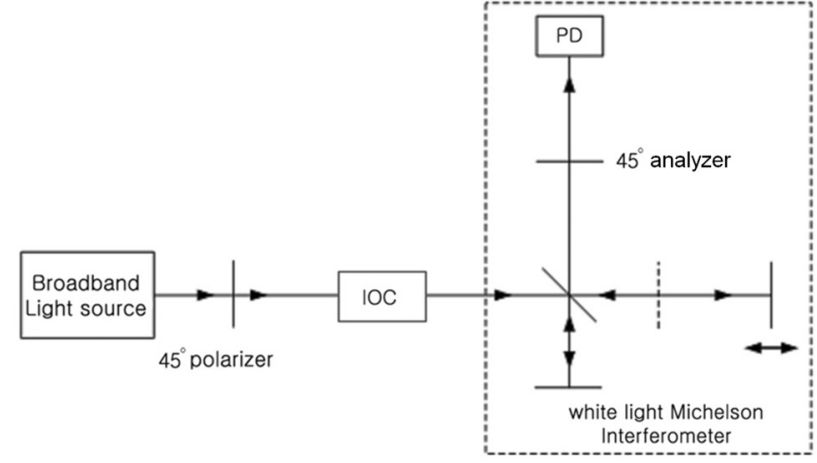

FIG. 2. Polarization measurement setup based on PM-OCDP.

than the analyzer, it is impossible to detect such a low value by investigating only the intensity ratio passing thorough the analyzer. Intensity extinction coefficient of a general polarizer such as a Glan-Thompson prism is around $-50 \mathrm{~dB}$, but the IOC is expected to have lower value less than $-60 \mathrm{~dB}$, therefore the fundamental difficulties could be prominent during the measurements.

On the other hand, polarization measurements based on PM-OCDP give a good solution for reading out the location and magnitude of polarization cross-coupling or extinction in the IOC precisely and simultaneously [11-13]. In addition, because the readout quantities in this method are not intensity but amplitude coefficient, it is possible to measure even very low values near the noise floor considered in the measurement system. A schematic view of a polarization measurement setup based on PM-OCDP is shown Fig. 2. A linearly-polarized optical wave at $45^{\circ}$ from polarization principal axes is launched into the IOC after passing through the input polarizer. As the lightwave is propagating along the IOC with two pigtailed PMFs and a proton exchanged $\mathrm{LiNbO}_{3}$ waveguide, input field is formed as spatially distributed optical wave-train caused by polarization cross coupling and polarization mode dispersion (PMD). After passing through the output pigtailed PMF, the formed wavetrains are divided identically as $50: 50$ by beam splitter and sent to two arms of the Michelson interferometer. One arm is mounted on a linear motor stage to change the position of the mirror along the propagation direction so that the birefringence path imbalance can be compensated. After experiencing the optical path between the beam splitter and the mirror, the two lightwaves are sent to the analyzer at $45^{\circ}$ from the polarization principle axes to superimpose the amplitudes in different states of polarization. Finally, the intensity of the superimposed lightwave is obtained at the photo detector. 


\section{THEORETICAL ANALYSIS OF PM-OCDP BASED ON JONES MATRIX REPRESENTATION}

Measured information from the PM-OCDP is basically that of spatially distributed optical wavetrains due to the PMD of the birefringent waveguides and local polarization cross-couplings. So it is necessary to perform additional activities to extract the correct values of $\rho_{r}$ and $\varepsilon_{\text {chip }}$ from the measured information. Furthermore, if the measured information can be overestimated somewhat by the limits on the performance of components used or by alignment error during the measurements, these realistic problems should be taken into consideration to obtain the true values of $\rho_{r}$ and $\varepsilon_{c h i p}$. Therefore, the theoretical estimation and analysis of PM-OCDP are essential prior to the measurements. To our knowledge, it is the first evaluation of the critical factors of IOC by applying the theoretical analysis based on Jones matrix representation to the measured interference pattern of PM-OCDP. A schematic diagram of PM-OCDP with IOC is shown in Fig. 3. The input light-wave source with an arbitrary degree of polarization (DOP) before the input polarizer can be expressed as

$$
E_{i n}=\left[\begin{array}{l}
E_{x}(t) \\
E_{y}(t)
\end{array}\right]=\left[\begin{array}{l}
a e_{x}(t) \\
b e_{y}(t)
\end{array}\right] e^{i \omega_{c} t}
$$

where $\omega_{c}$ is the source center frequency, $E_{x}(t)$ and $\mathrm{E}_{y}(t)$ are the polarization components of the input lightwave corresponding to $\mathrm{x}$ and $\mathrm{y}$ axes, $a e_{x}(\mathrm{t})$ and $b e_{y}(\mathrm{t})$ are complex amplitudes, $a$ and $b$ are real numbers [14-15]. In this study, the $\mathrm{x}$-direction is the main polarization axis corresponding to slow-mode in PMF and transverse electric field (TE) mode in IOC chip. When the source intensity is normalized as unity $\left(a^{2}+b^{2}=1\right)$, the source DOP, $P$ is defined as follows

$$
\boldsymbol{P}=\left|2 \boldsymbol{a}^{2}-1\right|
$$

The Jones matrix describing the lightwave coupled to input PMF after passing through the input polarizer can be expressed as follows

$$
\boldsymbol{E}_{o}=\boldsymbol{T}_{p o l}(\boldsymbol{\alpha}) \cdot \boldsymbol{E}_{i}=\left[\begin{array}{cc}
\cos \boldsymbol{\alpha} & -\sin \boldsymbol{\alpha} \\
\sin \boldsymbol{\alpha} & \cos \boldsymbol{\alpha}
\end{array}\right]\left[\begin{array}{cc}
1 & 0 \\
\delta & \boldsymbol{\varepsilon}_{p o l}
\end{array}\right] \boldsymbol{E}_{i}
$$

where the input polarizer is counterclockwise rotated by $\alpha$ degrees with respect to the main polarization axis of input PMF. And $\varepsilon_{p o l}$ is amplitude extinction coefficient and $\delta$ is scattering-related term of imperfect input polarizer. The scattering-related term allows scattering with $90^{\circ}$ polarization rotation of the incident field. In this study, the very low $\delta$ of the imperfect polarizer is assumed to be zero for simplicity as described in $[4,15]$. The Jones matrix explains the phase shift due to pigtailed input/output PMFs is [16]

$$
\boldsymbol{E}_{o}=\boldsymbol{T}_{\text {fiber }} \cdot \boldsymbol{E}_{i n} \cong\left[\begin{array}{cc}
\boldsymbol{e}^{-2 i m m_{t_{y}} d_{f} / \lambda} & 0 \\
0 & \boldsymbol{e}^{-2 i m t_{y f} d_{f} / \lambda}
\end{array}\right] \boldsymbol{E}_{i}
$$

where $n_{x f}, n_{y f}$ are the effective indexes of PMF corresponding to $\mathrm{x}, \mathrm{y}$ direction, $d_{f}$ is the length of the PMF and $\lambda$ means the wavelength of the optical wave. In the pigtailed PMF, we assume that there are no more polarization mode coupling points anywhere along the PMF. The transfer matrix for the local polarization cross-coupling, $\rho_{r}$, due to axis misalignment between PMF and the birefringent waveguide of the IOC chip is given by

$$
\boldsymbol{E}_{o}=\boldsymbol{T}_{\text {crosstalk }}\left(\rho_{r}\right) \cdot \boldsymbol{E}_{i}=\left[\begin{array}{cc}
\sqrt{1-\rho_{r}^{2}} & \rho_{r} \\
-\rho_{r} & \sqrt{1-\rho_{r}^{2}}
\end{array}\right] \boldsymbol{E}_{i}
$$

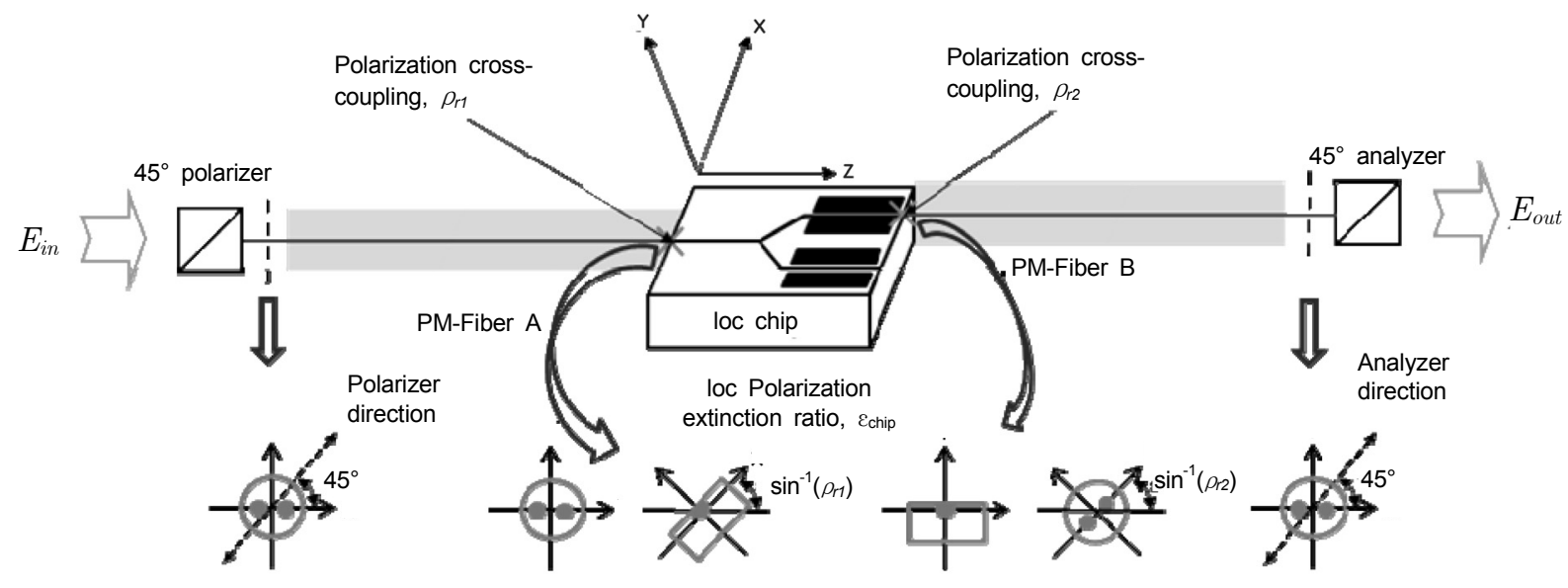

FIG. 3. Schematic diagram of PM-OCDP with IOC. 
For the case of the IOC chip, because there is also polarization rejection characteristic except for birefringence, the transfer matrix for the IOC chip can be expressed as product form.

$$
\boldsymbol{E}_{o}=\boldsymbol{T}_{c h i p} \cdot \boldsymbol{E}_{i n} \cong\left[\begin{array}{cc}
\boldsymbol{e}^{-2 i m m_{x c} d_{c} / \lambda} & 0 \\
0 & \boldsymbol{e}^{-2 i m_{y_{c} d_{c}} / \lambda}
\end{array}\right]\left[\begin{array}{cc}
1 & 0 \\
0 & \boldsymbol{\varepsilon}_{c h i p}
\end{array}\right] \boldsymbol{E}_{\boldsymbol{i}}
$$

where $n_{x c}, n_{y c}$ are effective indexes of birefringent IOC waveguide corresponding to $\mathrm{x}$, $\mathrm{y}$ directions, $d_{c}$ is the physical length of the IOC waveguide and $\varepsilon_{c h i p}$ is amplitude extinction coefficient of IOC chip. Eq. 8 is also valid under the assumption that there are no more polarization mode coupling points anywhere along the IOC waveguide. And the transfer matrix represents the change of polarization after passing through the analyzer counterclockwise rotated by $\beta$ degrees with respect to the main polarization axis of output PMF is given by similarly with Eq. 5,

$$
\boldsymbol{E}_{o}=\boldsymbol{T}_{\text {ana }}(\boldsymbol{\beta}) \cdot \boldsymbol{E}_{\text {in }}=\left[\begin{array}{cc}
1 & 0 \\
0 & \boldsymbol{\varepsilon}_{\text {ana }}
\end{array}\right]\left[\begin{array}{cc}
\cos \boldsymbol{\beta} & \sin \boldsymbol{\beta} \\
-\sin \boldsymbol{\beta} & \cos \boldsymbol{\beta}
\end{array}\right] \boldsymbol{E}_{\boldsymbol{i}}
$$

where $\varepsilon_{a n a}$ is amplitude extinction coefficient of the output analyzer.

Combining each analysis, the output lightwave passing through the entire IOC with two local polarization cross-couplings, $\rho_{r 1}$ and $\rho_{r 2}$ can be expressed as follows

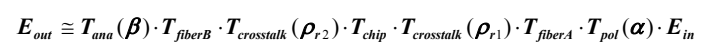

Therefore for the transit time difference, $\tau$ originated by the path differences from the beam splitter to two different arms of the Michelson interferometer, the interference output amplitude is given by

$$
E_{s} \cong \frac{1}{\sqrt{2}}\left[E_{\text {out }}(t)+E_{\text {out }}(t-\tau)\right]
$$

And the output intensity that can be obtained at the photo-detector is

$$
\boldsymbol{I} \cong<\boldsymbol{E}_{s} \cdot \boldsymbol{E}_{s}^{*}>
$$

where $<>$ signifies time average.

As $\tau$ is varied, the output intensity is changed by the polarization characteristics of the IOC. Inversely, this means that it can be possible to analyze the polarization characteristics from the measured output intensity. Let's consider the special case of $\alpha=\beta=45^{\circ}$ and $\varepsilon_{p o l}=\varepsilon_{\text {ana }}=0$ to understand the polarization measurement based on PM-OCDP. The $E_{\text {out }}$ for this case is

$$
\begin{aligned}
& \boldsymbol{E}_{\text {out }}\left(\boldsymbol{\alpha}=\boldsymbol{\beta}=45^{\circ} \mid \boldsymbol{\varepsilon}_{\text {pot }}=\boldsymbol{\varepsilon}_{\text {ana }}=0\right)
\end{aligned}
$$

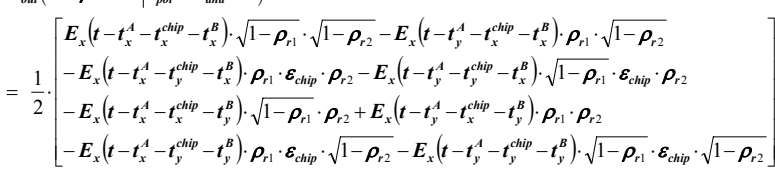

where $t_{x}^{A}$ represent transit time of the principle x-polarized mode in a waveguide A. As seen in Eq. 13, the optical wave passing through the analyzer is formed as eight spatially distributed optical wavetrains. Generally, because $\rho_{r 1}$ and $\rho_{r 2}$ due to polarization axis misalignments are low values below 0.1 , the first term in Eq. 13 has a maximum and this primary wavetrain serves as a local oscillator for the coherent detection of the other seven terms in Eq. 13. The seven parasitic wavetrains result from the two local polarization crosscouplings and contain the critical information about $\rho_{r}$ and $\varepsilon_{c h i p}$. The output intensity has a maximum at $\tau=0$ and the normalized modulation term of the interference can be approximated for $\tau>0$ as

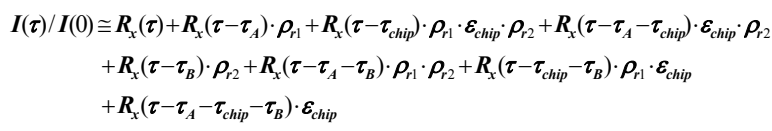

where $\mathrm{R}_{\mathrm{x}}(\tau)$ is the normalized autocorrelation function of the optical source, $R_{x}(\tau)=<E_{x}(t) E_{x}^{*}(t+\tau)>/<E_{x}(t) E_{x}^{*}(t)>$, and $\tau_{m}$ is the group delay difference between two principle polarization modes in $\mathrm{m}$ optical waveguide, $t_{x}^{m}-t_{y}^{m}$. If their longitudinal separation along the IOC is greater than the depolarization length, it is possible to differentiate the parasitic wavetrains due to local polarization cross-couplings. In this case, the two local polarization cross-couplings, $\rho_{1}$ and $\rho_{2}$ correspond to the values at $\tau=\tau_{A}$ and $\tau=\tau_{B}$, the amplitude extinction coefficient of the IOC chip correspond to the value at $\tau=\tau_{A}+\tau_{B}+\tau_{\text {chip }}$.

\section{THEORETICAL AND ANALYZED RESULTS}

In this chapter, the theoretical and analyzed results on measurements of the polarization characteristics of IOC based on OCDP are presented. In the process of theoretical estimation of the interference pattern of PM-OCDP, the spectral profile of the optical source is necessary to calculate the autocorrelation function. And DOP of the source also must be known for theoretical estimation. The power spectrum of the optical source is shown in Fig. 4. The optical source used is Erbium Doped Fiber Source (EDFS) with 2\% of DOP 


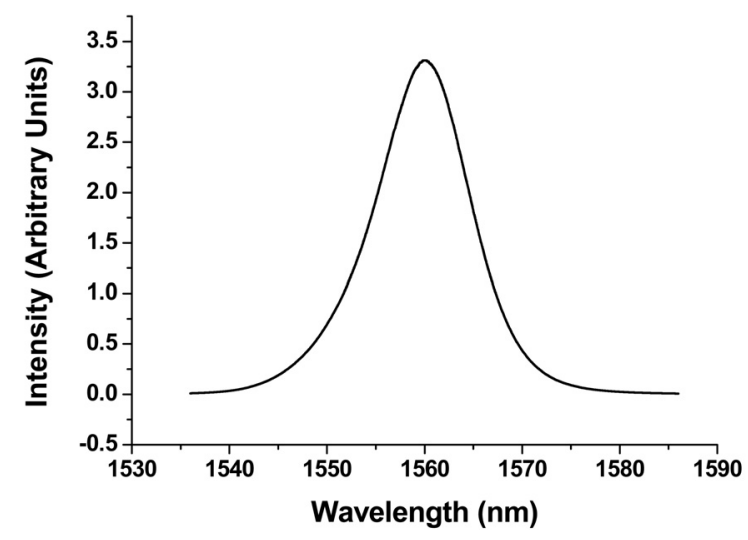

FIG. 4. Power spectrum of EDFS.

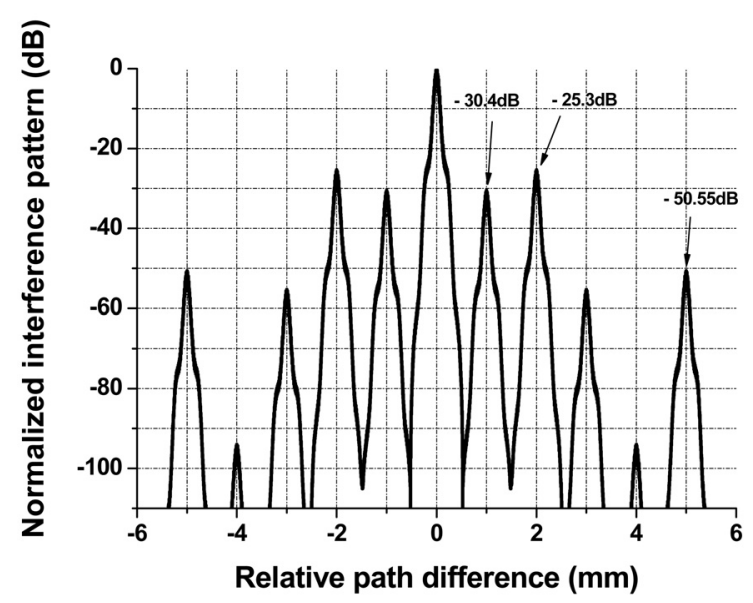

FIG. 5. Calculated interference pattern of PM-OCDP.

and $12 \mathrm{~nm}$ of full width at the half maximum (FWHM) at C-band. In Fig. 5, the calculated interference pattern of PM-OCDP is presented as a function of relative optical path difference between the fixed and moving mirror. The parameters used in the calculation are $P=0.02, \rho_{r 1}=-25.62 \mathrm{~dB}, \rho_{r 2}=-29.14 \mathrm{~dB}, \alpha=44^{\circ}, \beta$ $=46^{\circ}, \varepsilon_{c h i p}=50 \mathrm{~dB}, \varepsilon_{p o l}=\varepsilon_{a n a}=-30 \mathrm{~dB}, d_{f A}=4 \mathrm{~m}, d_{f B}=2 \mathrm{~m}$, $d_{c}=37.5 \mathrm{~mm}, \Delta_{n f}=5 \times 10^{-4}$ and $\Delta n_{c h i p}=0.057$. The $\Delta n_{f}$ and $\Delta n_{\text {chip }}$ signify the birefringence of PMF and proton exchanged $\mathrm{LiNbO}_{3}$ optical waveguide, respectively. Nonzero $\mathrm{P}, \varepsilon_{p o l}, \varepsilon_{a n a}$ and non $45^{\circ} \alpha, \beta$ are selected to take the real situation of measurement into consideration. Because the $\tau$ between the wavetrains are compensated by varying the optical path difference, it is noteworthy to express the interference pattern in terms of $\tau$. The $\tau$ of OCDP can be expressed in terms of birefringence as follows [17]

$$
\tau_{O C D P}=\frac{\partial \Delta \phi}{\partial \omega}=\frac{\partial \Delta \beta \boldsymbol{l}}{\partial \omega}=\frac{l}{c}\left[\Delta \boldsymbol{n}-\lambda \frac{\partial \Delta \boldsymbol{n}}{\partial \lambda}\right]
$$

Where $\beta$ is the propagation constant and $l$ signifies

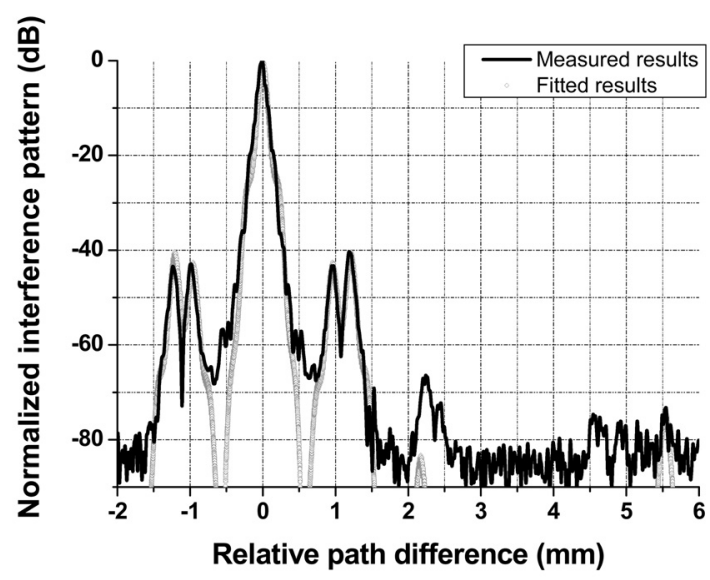

FIG. 6. Measured and fitted interference pattern of PM-OCDP (Sample No.1).

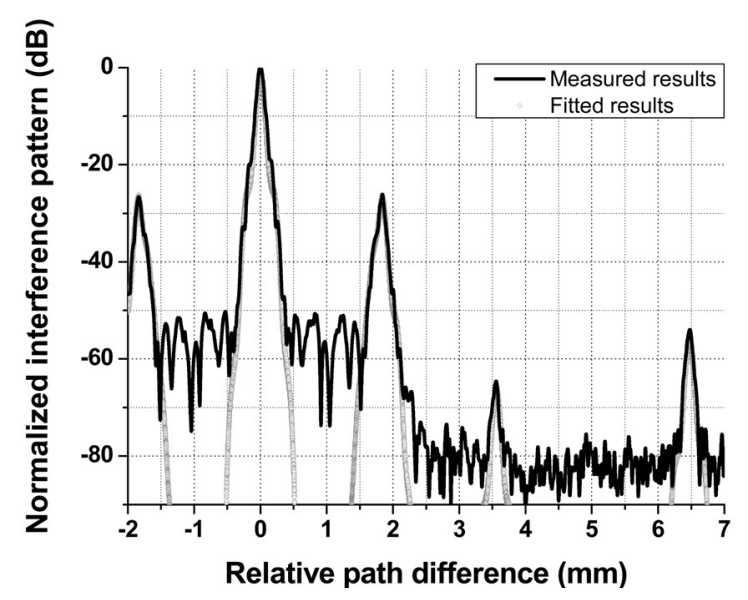

FIG. 7. Measured and fitted interference pattern of PM-OCDP (Sample No.2).

physical length of the optical waveguide being considered. The optical path difference $(L)$ at horizontal axis of Fig. 6 is generated under the assumption of that there is negligible effect of dispersion term, $\partial \Delta n / \partial \lambda$, in Eq. 15 as follows

$$
L=c \cdot \tau_{O C D P} \approx \Delta n l
$$

Therefore, the calculated values at $L= \pm 2 \mathrm{~mm}$, and $L=$ $\pm 1 \mathrm{~mm}$ correspond to $\rho_{r 1}$ and $\rho_{r 2}$, respectively. Similarly, the calculated value at $L= \pm 5 \mathrm{~mm}$ is corresponding to $\varepsilon_{c h i p}$ because the optical path difference of $5 \mathrm{~mm}$ is formed by the compositive PMD of IOC with pigtailed PMFs, $\tau_{O C D P}=\tau_{A}+\tau_{B}+\tau_{\text {chip }}$. From the calculated values shown in Fig. 5, it is found that there are small differences less than $1 \mathrm{~dB}$ between the pre-assumed and calculated values. These small discrepancies are caused by non-ideal measurement conditions, non zero of $P$, $\varepsilon_{p o l}, \varepsilon_{a n a}$ and non $45^{\circ} \alpha, \beta$. 
TABLE 1. Summary of analyzed results

\begin{tabular}{c|c|c|c|c}
\hline \hline Sample No. & $\rho_{r 1}^{2}(\mathrm{~dB})$ & $\rho_{r 2}^{2}(\mathrm{~dB})$ & $\varepsilon_{\text {chip }}^{2}(\mathrm{~dB})$ & $\Delta n_{\text {chip }}$ \\
\hline $\begin{array}{c}1 \\
\left(d_{f A}=1.9 \mathrm{~m}, d_{f B}=2.4 \mathrm{~m}\right)\end{array}$ & -43.12 & -39.60 & -73 & 0.089 \\
\hline $\begin{array}{c}2 \\
\left(d_{f A}=3.7 \mathrm{~m}, d_{f B}=3.45 \mathrm{~m}\right)\end{array}$ & -26.54 & -38.26 & -54 & 0.078 \\
\hline
\end{tabular}

The measured and theoretically fitted interference patterns of PM-OCDP for two IOC samples are presented in Fig. 6 and 7. Because of the noise level around $-90 \mathrm{~dB}$ in the measurement system, there is no information under $-90 \mathrm{~dB}$. In Fig. 7, an additional interference peak near $L=2.2 \mathrm{~mm}$ and higher values near $L=0.6 \mathrm{~mm}$ in measured results is shown as comparing with fitted results. These discrepancies seem to be presented by another factor not related to polarization characteristics, such as optical reflections generated along the in-line connection of the measurement system. For the case of interference pattern shown in Fig. 8, there is quite a different shape near the location at $L=1.78 \mathrm{~mm}$ as compared with typical shape shown in Fig. 6 or 7 . It is because that two wave amplitudes representing $\rho_{r 1}$ and $\rho_{r 2}$ are in superposition caused by small length difference between input and output PMF. Even though the measured results are slightly different from the typical shape as shown in Fig. 5, the critical factors $\rho_{r 1}, \rho_{r 2}$ and $\varepsilon_{c h i p}$ could be extracted accurately by applying theoretical fitting process to the measured interference pattern. In addition, birefringence of proton exchanged $\mathrm{LiNbO}_{3}$ optical waveguide was also analyzed from Eq. 16. All analyzed results are summarized in Table 1.

\section{CONCLUSION}

Accurate evaluation of polarization characteristics in IOC for IFOG was suggested theoretically and confirmed. Critical factors that determine bias stability of IFOG such as local polarization cross-couplings or polarization extinction coefficient of IOC were identified accurately by applying theoretical analysis based on Jones matrix representation into the measured interference pattern of PM-OCDP. Our evaluation approach provides a very useful tool for analyzing the birefringence of IOC waveguide and characterizing the error factors of the polarization measurement utilizing PM-OCDP.

\section{REFERENCES}

1. R. A. Bergh, H. C. Lefervre, and H. J. Shaw, "An overview of fiber-optic gyroscopes," J. Lightwave Technol. 2, 91-107 (1984).

2. R. Ulrich, "Fiber-optic rotation sensing with low drift," Opt. Lett. 5, 173-175 (1980).

3. R. Ulrich, "Fiber-ring interferometer: polarization analysis," Opt. Lett. 4, 152-154 (1979).

4. E. C. Kintner, "Polarization control in optical-fiber gyroscopes," Opt. Lett. 6, 154-156 (1981).

5. R. A. Bergh, H. C. Lefevre, and H. J. Shaw, "All-singlemode fiber-optic gyroscope with long-term stability," Opt. Lett. 6, 502-504 (1981).

6. E. Jones and J. W. Parker, "Bias reduction by polarisation dispersion in the fibre-optic gyroscope," Electron. Lett. 22, 54-56 (1986).

7. H. C. Lefervre, The Fiber-optic Gyroscope (Artech House, Boston, MA, USA, 1993), Chapter 3.

8. H. C. Lefervre, J. P. Bettini, S. Vatoux, and M. Papuchon, "Progress in optical fiber gyroscoeps using integrated optics," Proc. SPIE MS 8, 216-227 (1985).

9. Y. N. Korkishko, V. A. Fedorov, and O. Y. Feoktistova, " $\mathrm{LiNbO}_{3}$ optical waveguide fabrication by high-temperature proton exchange,” J. Lightwave Technol. 18, 562-568 (2000).

10. S. C. Rashleigh, W. K. Burns, R. P. Moeller, and R. Ulrich, "Polarization holding in birefringent single-mode fibers," Opt. Lett. 7, 40-42 (1982).

11. H. C. Lefervre, "Comments about fiber-optic gyroscopes,” Proc. SPIE 838, 86-97 (1987).

12. K. Takada, J. Noda, and K. Okamoto, "Measurement of spatial distribution of mode coupling in birefringent polarization-maintaining fiber with new detection scheme," Opt. Lett. 11, 680-682 (1986).

13. K. Takada, K. Chida, and J. Noda, "Precise method for angular alignment of birefringent fiber based on interferometric technique,” Appl. Opt. 26, 2979-2987 (1987).

14. W. K. Burns and R. P. Moeller, "Polarizer requirements for fiber gyroscopes with high-birefringence fiber and broad-band sources," J. Lightwave Technol. 2, 430-435 (1984).

15. W. K. Burns, "Phase error bounds of fiber gyro with polarization holding fiber," J. Lightwave Technol. 4, 8-14 (1986).

16. H. C. Lefervre, The Fiber-optic Gyroscope (Artech House, Boston, MA, USA, 1993), Appendix 1.

17. K. Okamoto, Fundamentals of Optical Waveguides (Academic Press, New York, NY, USA, 2000), Chapter 3. 